\title{
Evaluation of Sputum Decontamination Methods to Facilitate the Mycobacterium tuberculosis Detection in a Tertiary Care Hospital
}

\author{
R. Someshwaran*, Shreeram. A. Deshpande and K. Gnanaprakash \\ Department of Microbiology, Karpagam Faculty of Medical Sciences and Research, \\ Othakkalmandapam, Coimbatore-641032, Tamil Nadu, India \\ *Corresponding author
}

\begin{tabular}{l} 
Ke y w o r d s \\
Mycobacterium \\
tuberculosis, \\
Sputum samples, \\
for Hypertonic \\
Saline - Sodium \\
Hydroxide \\
Method. \\
\hline Article Info \\
\hline \\
Accepted: \\
28 July 2016 \\
Available Online: \\
10 August 2016
\end{tabular}

\section{A B S T R A C T}

Tuberculosis caused by Mycobacterium tuberculosis (or Koch's bacillus) even though curable and preventable it is still one of the leading infectious causes of morbidity and mortality especially in developing countries like India. Diagnostic methods available to detect tuberculosis are Direct approach by Microscopy, Culture, Biochemical test, Nuclear techniques. In developing countries diagnosis of acid fast bacilli is performed by microscopic examination of Ziehl-Neelsen (ZN) stained sputum smear, because it is simple, inexpensive and it provides rapid results. But this technique has a low sensitivity $22-43 \%$ for single smear. And also up to $60 \%$ sensitivity under optimal conditions, when compared with cultures so that culture remains as gold standard to diagnose tuberculosis. The threshold for detection of acid fast bacilli in sputum samples under optimal condition is between $10^{4}-10^{5}$ bacilli $/ \mathrm{ml}$, which can be obtained by decontamination \& concentration techniques. Sputum microscopy remains the mainstay of diagnosis of pulmonary tuberculosis but lacks sensitivity compared to culture. Pre-requisite for microscopic examination of sputum sample is prior decontamination. Rationale for the study: There are only a very few studies done in South India to report that Hypertonic Saline Sodium Hydroxide (HS-SH) method of decontamination is superior to gold standard NALC-NaOH method in India. So, we would like to evaluate Hypertonic Saline Sodium Hydroxide (HS-SH) method for decontamination of sputum samples for sputum microscopy. Main objective of our study is to evaluate the performance of the Novel Hypertonic Saline Sodium hydroxide (HS-SH) method with the gold standard NALC-NaOH for decontamination of sputum specimens to detect acid fast Mycobacterium tuberculosis. Institutional Human Ethical Committee clearance and Patient's informed written consent obtained. The Study was conducted as a prospective epidemiological study in Diagnostic Microbiology section of Central Service Laboratory for 6 months from November 11, 2015 to May 10, 2016. Total of 143 sputum samples obtained from both in-patients and out-patients of Karpagam Medical College Hospital from various departments like Medicine, Surgery, TB \& RD and Orthopedics were subjected to decontamination by both HS-SH method and NALC-NAOH methods. Slides were then examined under oil immersion for acid fast bacilli by conventional Ziehl - Neelsen staining microscopic method. Results: Out of 143 microbiological sputum samples, 104 were found to be positive for acid fast bacilli by both Modified petroff's method and Hypertonic Saline Sodium Hydroxide method (HS-SH) of concentration and decontamination and 39 samples were negative by both these methods. Sensitivity, Specificity, Positive predictive value \& Negative predictive value of the HS-SH method was found to be $100 \%$. Conclusion: The HS-SH sputum decontamination method is equally sensitive, specific, cost-effective, feasible and less time consuming procedure compared to the gold standard NALC-NaOH method for decontamination of Mycobacterium tuberculosis from sputum samples and can be routinely used in all peripheral health centres because of the low cost, especially in developing countries. 


\section{Introduction}

Tuberculosis caused by Mycobacterium tuberculosis (or Koch's bacillus) even though curable and preventable it is still one of the leading infectious causes of morbidity and mortality especially in developing countries like India. Global incidence of tuberculosis is 9.4 million cases annually, whereas India accounts for 2.0 million i.e., one-fifth of the Global incidence (2010). The Global prevalence rate is 3.8 million bacteriologically positive cases (2000) with death rate of 3, 25,000 per year due to TB. A total of 8.6 million patients newly developed tuberculosis in 2012 as estimated accounting for a mortality of 1.3 million cases (Global Tuberculosis report, World Health Organization, 2013). In India the prevalence rate is 249 per lakh population (2009). The life time risk of a person developing Tuberculosis is $10 \%$ (10 out of 100$)$. Diagnosis of Tuberculosis is by a combination of proper history taking, clinical examination, Microbiological evaluation by Sputum microscopy and Culture (Conventional or automated) which is the gold standard and also by correlation with roentgenologic examination. Resistance in Tuberculosis had become a global menace for treating physicians. Diagnostic methods available to detect tuberculosis are (ICMR Bulletin, 2002): Direct approaches are by Microscopy, Culture, Biochemical test, Nuclear techniques. In developing countries diagnosis of acid fast bacilli is performed by microscopic examination of Ziehl-Neelsen (ZN) stained sputum smear, because it is simple, inexpensive and it provides rapid results. But this technique has a low sensitivity $22-43 \%$ for single smear (Toman, 2004). And also up to $60 \%$ under optimal conditions (Asper et al., 2003), when compared with cultures so that culture remains as gold standard to diagnose tuberculosis. The threshold for detection of acid fast bacilli in sputum samples under optimal condition is between $10^{4}-10^{5}$ bacilli/ml, which can be obtained by decontamination \& concentration techniques (IUATLD, 2005).

Sputum microscopy remains the mainstay of diagnosis of pulmonary tuberculosis but lacks sensitivity compared to culture. Prerequisite for microscopic examination of sputum sample is prior decontamination. Conventional methods for decontamination include Petroff's method which is routinely done, while N-Acetyl-L-Cysteine Sodium Hydroxide (NALC-NaOH) still remains a gold standard. N- Acetyl -L- Cysteine method has $4 \% \mathrm{NaOH}$ and kills $60-70 \%$ of Mycobacteria, and the sensitivity for acid fast smear is $66.7 \%$. Hypertonic SalineSodium Hydroxide (HS-SH) method has only $1.33 \% \quad \mathrm{NaOH}$ is used for decontamination (Ganoza et al., 2008). Concentrated sputum techniques are much better than direct sputum microscopy and Modified Petroff's method is the widely used DC method but this method is too cumbersome to carry in field locations (Vasanthakumari et al., 1988). Various decontamination methods used for decontamination of sputum samples for Mycobacterium tuberculosis are Bleach (6\% $\mathrm{NaOCl}$ ) method, Petroff's method, Modified Petroff's Sodium Hydroxide method, NAcetyl-L-Cysteine- sodium Hydroxide method (NALC - NaOH), Hypertonic Saline Sodium Hydroxide(HS-SH) method, Zephiran- Trisodium Phosphate (Z-TSP) method, Oxalic Acid method, Sulphuric Acid method, Cetyl-Pyridinium Chloride Sodium chloride (CPC) method and Phenyl Ammonium sulphate (Ricaldi et al., 2008; Singhal et al., 2013). Relative Centrifugal Force for HS-SH method is high (4000rpm) and the sensitivity for acid fast bacilli smear is about $71.4 \%$ which is more than the $\mathrm{N}$ acetyl-L-cysteine-Sodium hydroxide decontamination and concentration method. 
In hypertonic saline- $\mathrm{NaOH}$ method, hypertonic saline is used for mucolysis and Sodium hydroxide for decontamination (King et al., 1997). Literature survey has shown HS-SH technique substantially increased the sensitivity of AFB smears compared with the routine direct smear for AFB and correlated well with the NALC$\mathrm{NaOH}$ decontamination method for sputum culture in suspected Tuberculosis patients (King et al., 1997; Ricaldi et al., 2008). Hypertonic saline has been used successfully for the symptomatic treatment of cystic fibrosis. There are only a very few studies to report that Hypertonic Saline Sodium Hydroxide (HS-SH) method of decontamination is superior to gold standard NALC-NaOH method in India. So, we would like to evaluate Hypertonic Saline Sodium Hydroxide (HS-SH) method for decontamination of sputum samples for sputum microscopy.

The main objectives of this study to evaluate the performance of the Novel Hypertonic Saline Sodium hydroxide (HS-SH) method with the gold standard NALC-NaOH for decontamination of sputum specimens to detect acid fast Mycobacterium tuberculosis.

\section{Materials and Methods}

Institutional Human Ethical Committee clearance and Patient's informed written consent obtained. The Study was conducted as a prospective epidemiological study in Diagnostic Microbiology section of Central Service Laboratory for 6 months from November 11, 2015 to May 10, 2016. Total of 143 sputum samples obtained from both in-patients and out-patients of Karpagam Medical College Hospital from various departments like Medicine, Surgery, TB\&RD and Orthopedics were subjected to decontamination by both HS-SH method and NALC-NAOH methods. Slides were then examined under oil immersion for acid fast bacilli by conventional Ziehl-Neelsen staining microscopic method.

\section{Inclusion Criteria}

1. Patients with cough more than 2 weeks and/or blood stained sputum.

2. New sputum positive pulmonary tuberculosis patients before initiation of treatment.

\section{Exclusion Criteria}

1. Patient already under anti-tubercle drug treatment.

2. Inadequate sample volume $(<2 \mathrm{ml})$.

3. Sample not representative of lower respiratory tract.

4. Patient denying participating in the study.

\section{Decontamination Method}

Procedure for Hypertonic Saline - Sodium Hydroxide Method (HS-SH) (Singhal et al., 2013; Nora Morcillo et al., 2008):

One $\mathrm{ml}$ portion of sputum was mixed with one $\mathrm{ml} 7 \%(\mathrm{w} / \mathrm{v}) \mathrm{NaCl}$ and one $\mathrm{ml} 4 \%$ $(w / v) ~ \mathrm{NaOH}$ in a sterile $15 \mathrm{ml}$ centrifuge tube (BD Falcon) and homogenized for 1520 s using a vortex mixer [Final concentrations (w/v) in $3 \mathrm{ml}: 2.33 \% \mathrm{NaCl}$, $1.33 \% \mathrm{NaOH}]$. The tubes were then incubated at $37^{\circ} \mathrm{C}$ for $30 \mathrm{~min}$. After incubation, the mixture was neutralized with sterile PBS $(\mathrm{pH}$ 6.8), bringing the total volume to $15 \mathrm{ml}$. The mixture was vortexed for $5 \mathrm{~s}$ and then centrifuged at $3400 \mathrm{~g}$ for 15 min at $15^{0} \mathrm{C}$, using aerosol proof shields. The supernatant was discarded into a splashproof container with a tuberculocidal solution. The procedure used has been described previously and is recommended by the Centers for Disease Control and Prevention and the WHO/IUATLD. Briefly, 
one $\mathrm{ml}$ sputum was added to a $50 \mathrm{ml} \mathrm{BD}$ Falcon centrifuge tube with one $\mathrm{ml}$ of solution containing $0.5 \% \quad(\mathrm{w} / \mathrm{v})$ NALC, $2.67 \%(\mathrm{w} / \mathrm{v}) \mathrm{NaOH}$ and $1.45 \%(\mathrm{w} / \mathrm{v})$ sodium citrate and mixed well (final concentrations (w/v) in $2 \mathrm{ml}: 0.25 \%$ NALC, $1.34 \% \mathrm{NaOH}$, $0.73 \%$ sodium citrate). The tubes were incubated at room temperature for $15 \mathrm{~min}$. After incubation, the mixture was neutralized with PBS, bringing the total volume to $50 \mathrm{ml}$. The rest of the procedure was as described above.

\section{ZN sputum Smear preparation}

AFB sputum smear preparation done in Bio safety cabinet level II. One drop of each suspended pellet was used to prepare slides for AFB microscopy using the ZiehlNeelsen stain. Each slide was coded, read blindly by a qualified technician and reported according to the National Tuberculosis Program and WHO/IUATLD standards (WHO, 1998). Smears were reported as follows: Grade 0 where no Acid Fast Bacilli (AFB) is observed in a total of 200 oil immersion fields, Scanty (Sc) where 1-9 AFB in 100 microscopic fields (few bacilli) is observed; 1+ with 10-99 AFB in 100 fields; $2+$ with $1-10$ AFB per field in at least 50 fields; 3+ with more than 10 AFB per field in at least 20 fields. Each slide will be coded, examined and graded according to the RNTCP guidelines.

\section{Results and Discussion}

Out of 143 microbiological sputum samples, 104 were found to be positive for acid fast bacilli by both Modified petroff's method and Hypertonic Saline Sodium Hydroxide method (HS-SH) of concentration and decontamination and 39 samples were negative by both these methods. Sensitivity, Specificity, Positive predictive value \& Negative predictive value of the HS-SH method was found to be $100 \%$.

Table.1 Comparison of N-Acetyl -L- Cysteine Sodium Hydroxide (NALC-NaOH) and Hypertonic Saline Sodium Hydroxide (HS-SH) methods:

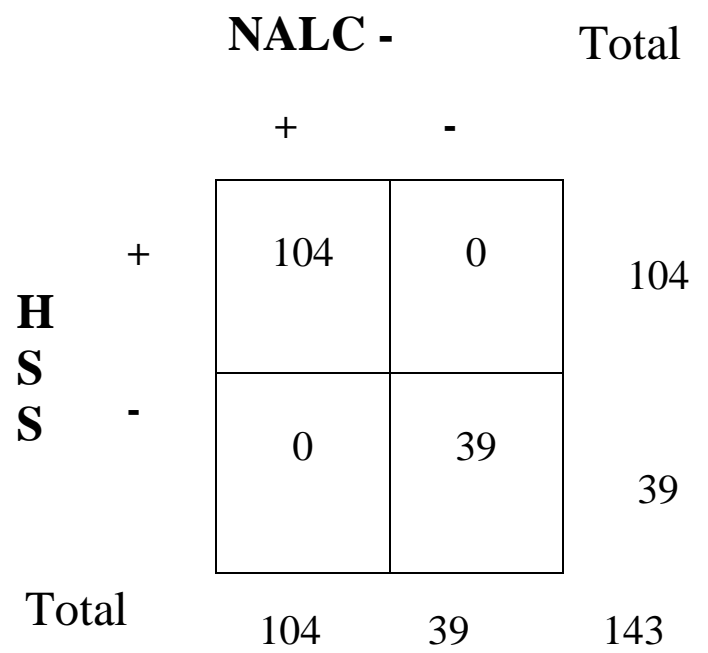

Decontamination methods for sputum microscopy for diagnosis of tuberculosis presently used are cumbersome, time consuming and expensive. Better methods 
are needed particularly developing countries. According to the study conducted by Christian Ganoza et al., the sensitivity for acid-fast bacilli (AFB) smears had increased from $28.6 \%$ using the direct method to 71.4\% Hypertonic Saline Sodium Hydroxide (HS-SH) method and $66.7 \%$ N-Acetyl-LCysteine (NALC-NaOH) using decontamination (DC) methods. In a study conducted in India, showed sensitivity of NALC-NaOH and HS-SH methods were $46 \%$ and $52 \%$ respectively (Sushil Kumar Chaudary, 2010). The proposed novel HSSH DC method improved the sensitivity of AFB microscopy compared with a routine direct smear; its performance was comparable to that of the Modified Petroff's method for AFB smears, but it was methodologically simpler and less expensive, equally sensitive and can be adopted in National TB control programme especially in developing countries.

Sensitivity, Specificity, Positive predictive value \& Negative predictive value of the HS-SH method was found to be $100 \%$ which is comparable with the standard Modified petroff's method. Novel HS-SH method was not all cumbersome compared to the gold standard NALC-NaOH method.

In conclusion, the HS-SH sputum decontamination method is equally sensitive, specific, cost-effective, feasible and less time consuming procedure compared to the gold standard NALC$\mathrm{NaOH}$ method for decontamination of Mycobacterium tuberculosis from sputum samples and can be routinely used in all peripheral health centres because of the low cost, especially in developing countries.

\section{References}

Global Tuberculosis report, World Health Organization, WHO Press, Geneva, Switzerland, 2013, pp.10-36.
Toman, K. 2004. Tuberculosis Case-Finding \& Chemotherapy. Case Detection, treatment and monitoring - Questions $\&$ Answers, $2^{\text {nd }}$ Edition Geneva WHO.

Asper, L., Mutsvangwa, J., Magwenzi, J., Chigara, N., Butterworth, A., Maso N, P. and Van der Stuyft, P. 2003. A comparison of direct microsopy, the concentration method and the Mycobacteria Growth Indicator Tube for the examination of sputum for acid fast bacilli. Ent. J. Tuberc. Lung Dis., 7, 376-381.

IUATLD. 2005. Tuberculosis bacteriologypriorities and indications in high prevalence countries: position of the technical staff of the Tuberculosis Division of the International Union Against Tuberculosis and Lung Disease. Int. J. Tuberculosis and Lung dis., 9, 355-361.

Ganoza, C.A., Ricaldi, J.N., Chauca, J., Rojas, G., Munayco, C., Agapito, J. et al. 2008. Novel hypertonic salinesodium hydroxide (HS-SH) method for decontamination and concentration of sputum samples for Mycobacterium tuberculosis microscopy and culture. J. Med. Microbiol.,57: 1094-98.

Vasanthakumari, et al. 1988. Concentrated sputum smear microscopy: A simple approach to better case detection in pulmonary tuberculosis. Indian $J$. Tuberculosis, 35: 80.

King, M., Dasgupta, B., Tomkiewiz, R.P. \& Brown, N.E. 1997. Rheology of cystic fibrosis sputum after in vitro treatment with hypertonic saline alone and in combination with recombinant human deoxyribonuclease I. Am. J. Respir. Crit. Care Med., 156, 173-177.

Ricaldi, J.N and Guerra, H. 2008. A simple and improved method for diagnosis of Tuberculosis using hypertonic saline and sodium hydroxide (HS-SH) to 
concentrate and decontaminate sputum. Trop. Doct., 38: 97-99.

Singhal, R., M. Bhalla, S. Chakraborty, D. Beheram V.P. Myneedu. 2013. Phenol1Ammonium sulfate microscopy method for diagnosis of pulmonary tuberculosis. Int. J. Mycobacteriol., 2: 84-88.

Nora Morcillo, Belén Imperiale, Juan Carlos Palomino; New simple decontamination method improves microscopic detection and culture of mycobacteria in clinical practice.
Infection and Drug Resistance, 1: 2126.

Kent, P.T., \& Kubica, G.P. 1985. 21-44.

Sushil Kumar Chaudary, Dept. of Microbiology, St. John's Medical college, Karnataka. 2010, "Comparison of Hypertonic Saline Sodium Hydroxide decontamination method with Modified Petroff's method for concentrating sputum samples"

ICMR Bulletin. 2002. Vol. 32, No.8, Part I: Techniques for diagnosis of T.B.

\section{How to cite this article:}

Someshwaran, R., Shreeram A. Deshpande and Gnanaprakash, K. 2016. Evaluation of Sputum Decontamination Methods to Facilitate the Mycobacterium tuberculosis Detection in a Tertiary Care Hospital. Int.J.Curr.Microbiol.App.Sci. 5(8): 879-894. doi: http://dx.doi.org/10.20546/ijcmas.2016.508.100 